\title{
Atorvastatin attenuates atherosclerotic plaque destabilization by inhibiting endoplasmic reticulum stress in hyperhomocysteinemic mice
}

\author{
FANG JIA $^{1}$, CHUNFANG WU ${ }^{2}$, ZHENYUE CHEN ${ }^{2}$, GUOPING LU ${ }^{2}$ and JIANHUI SUN ${ }^{1}$ \\ ${ }^{1}$ Department of Cardiology, The Third Affiliated Hospital of Soochow University, Changzhou, Jiangsu 213003; \\ ${ }^{2}$ Department of Cardiology, Ruijin Hospital, Shanghai Jiaotong University School of Medicine, Shanghai 200025, P.R. China
}

Received March 12, 2015; Accepted January 12, 2016

DOI: $10.3892 / \mathrm{mmr} .2016 .4975$

\begin{abstract}
Endoplasmic reticulum (ER) stress has been suggested to play a role in the progression of plaque vulnerability and the occurrence of acute complications of coronary atherosclerosis. Atorvastatin is known to exert pleiotropic effects on the cardiovascular system. The present study aimed to examine the stabilizing effects of atorvastatin on vulnerable plaques within hyperhomocysteinemic apolipoprotein E-deficient $\left(\mathrm{ApoE}^{-/-}\right)$mice, and to investigate the potential mechanisms underlying ER stress in $\mathrm{ApoE}^{-/-}$mice and macrophages. In the present study, $\mathrm{ApoE}^{-/-}$mice were administrated methionine or atorvastatin, and were sacrificed after 2 months. Necrotic core size, collagen content and inflammatory cytokine infiltration were subsequently measured in the aortic lesions, in order to investigate plaque stability. Treatment with atorvastatin decreased the number and size of necrotic cores, increased collagen content, and downregulated tumor necrosis factor (TNF)- $\alpha$ and matrix metalloproteinase (MMP)-9 mRNA expression, as compared with the methionine group. Immunohistochemical analysis indicated that atorvastatin administration prevented ER stress activation in aortic lesions of hyperhomocysteinemic mice. Furthermore, macrophages were challenged with homocysteine (Hcy) in the presence or absence of atorvastatin and thapsigargin (an ER stress inducer). Atorvastatin suppressed Hcy-induced ER stress, and downregulated TNF- $\alpha$ and MMP-9 mRNA expression in the macrophages. Conversely, thapsigargin attenuated
\end{abstract}

Correspondence to: Mr. Jianhui Sun, Department of Cardiology, The Third Affiliated Hospital of Soochow University, 185 Juqian Road, Changzhou, Jiangsu 213003, P.R. China

E-mail: jianhuisun_czyy@126.com

Mr. Guoping Lu, Department of Cardiology, Ruijin Hospital, Shanghai Jiaotong University School of Medicine, 197 Ruijin No. 2 Road, Shanghai 200025, P.R. China

E-mail: guopinglu_52@126.com

Key words: atorvastatin, homocysteine, endoplasmic reticulum stress, plaque stability the inhibitory effects of atorvastatin against Hcy-induced TNF- $\alpha$ and MMP-9 expression. These results indicated that hyperhomocysteinemia may promote atherosclerotic plaque development and instability. In addition, atorvastatin was able to improve atherosclerotic plaque stability in hyperhomocysteinemic mice by inhibiting ER stress.

\section{Introduction}

Atherosclerotic plaque destabilization and rupture are thought to account for the majority of acute coronary syndromes. Rupture-prone unstable plaques possess the following histological features: Lipid core formation, fibrous cap thinning, inflammatory cell infiltration of the fibrous cap and adventitia (1). Plaque destabilization may be induced by external factors, including increased blood pressure and shear stress, and/or by factors within the atherosclerotic plaque, including inflammation and intraplaque hemorrhage. Macrophages in atherosclerotic plaques have a marked impact on atherogenesis and plaque destabilization. During the development of atherosclerotic plaques, macrophages can initiate lesion progression, destabilization and rupture via the production and release of various cytokines and proteases, including tumor necrosis factor (TNF)- $\alpha$ and matrix metalloproteinases (MMPs) (2). Furthermore, exacerbated macrophage apoptosis may contribute to necrotic core expansion and fibrous cap thinning during advanced stages of the disease (3).

It has previously been suggested that endoplasmic reticulum (ER) stress may have a role in the progression of plaque vulnerability, and the occurrence of acute complications associated with coronary atherosclerosis (4). ER stress is chronically activated in atherosclerotic lesional cells, particularly in advanced lesional macrophages and endothelial cells. In vitro and in vivo studies have demonstrated that prolonged ER stress may result in macrophage-derived foam cell formation and proatherogenic cytokine expression in advanced atherosclerotic lesions $(5,6)$. Atherosclerotic-relevant inducers of ER stress, including modified forms of low-density lipoprotein (LDL) and hyperhomocysteinemia (HHcy), have an important role in inflammation and cell apoptosis in atherosclerotic lesions $(7,8)$. HHcy-induced ER stress has been reported to initiate and accelerate atherosclerosis via the 
upregulation of genes associated with lipid biosynthesis and uptake, inflammation, collagen synthesis and apoptosis (8). Due to its indispensable role in the progression of atherosclerosis, ER stress is therefore considered an important molecular target for the treatment of HHcy.

Atorvastatin, which is a 3-hydroxy-3-methylglutaryl coenzyme A reductase inhibitor, exerts antioxidant and anti-inflammatory functions independent of its lipid-lowering abilities. Atorvastatin has previously been shown to attenuate macrophage foam cell formation by regulating scavenger receptor expression, oxidized-LDL uptake and cholesterol efflux $(9,10)$. In addition, atorvastatin is able to alter the cytokine balance in the microenvironment of atherosclerotic plaques (11). In our previous studies, it was demonstrated that atorvastatin could antagonize homocysteine (Hcy)-induced endothelial dysfunction by increasing the viability of endothelial progenitor cells, and suppressing the apoptosis of endothelial cells $(12,13)$. To further define the underlying mechanisms by which atorvastatin inhibits HHcy-induced injury, the present study aimed to test the hypothesis that the plaque stabilizing effects of atorvastatin are partly attributed to inhibition of ER stress. Initially, we aimed to confirm whether atorvastatin was able to suppress ER stress activation in the vessel walls of hyperhomocysteinemic apolipoprotein E-deficient $\left(\mathrm{ApoE}^{-/}\right)$mice. Subsequently, the ability of atorvastatin to inhibit ER stress in macrophages was investigated.

\section{Materials and methods}

Animal experiments. The 6-week-old male ApoE ${ }^{-/}$mice were obtained from Peking University Health Science Center (Beijing, China). ApoE ${ }^{-/}$mice were chosen since they enabled the generation of a HHcy atherosclerotic model within 2 months, following the administration of $1 \mathrm{ml} 2 \%(\mathrm{w} / \mathrm{v})$ methionine (Sigma-Aldrich, St. Louis, MO, USA) per day by gastric gavage. The mice were housed in a temperature-controlled environment with a 12-h dark-light cycle and ad libitum access to food and water. The mice were divided into three groups: The methionine group, $\mathrm{ApoE}^{-/-}$mice were administered methionine $(n=15)$; the atorvastatin group, $\mathrm{ApoE}^{-/}$mice were administered atorvastatin $\left(5 \mathrm{mg} \cdot \mathrm{kg}^{-1} \cdot \mathrm{d}^{-1}\right.$; National Institute for the Control of Pharmaceutical and Biological Products, Beijing, China) suspended in $1 \mathrm{ml} \mathrm{2 \%}$ methionine $(n=15)$; and the control group, ApoE ${ }^{-/}$mice were administered saline $(n=20)$. After 2 months, the mice were anesthetized with $3 \%$ pentobarbital $(30 \mathrm{mg} / \mathrm{kg}$; China National Medicines Corporation, Ltd., Shanghai, China) and then sacrificed by exsanguination. The maximum amount of blood was obtained from the abdominal aorta and the intact circulation was flushed with phosphate-buffered saline (PBS). The aortic roots were carefully dissected and adherent connective tissue was removed. The animal experiments were conducted according to the recommendations of the Guidelines of National Animal Care and Use Committees, and the National Animal Welfare Law (14). The study was approved by the ethics committee of Soochow University (Suzhou, China).

Measurements of plasma Hcy. Following a 12-h fast and the induction of anesthesia, blood samples were obtained,

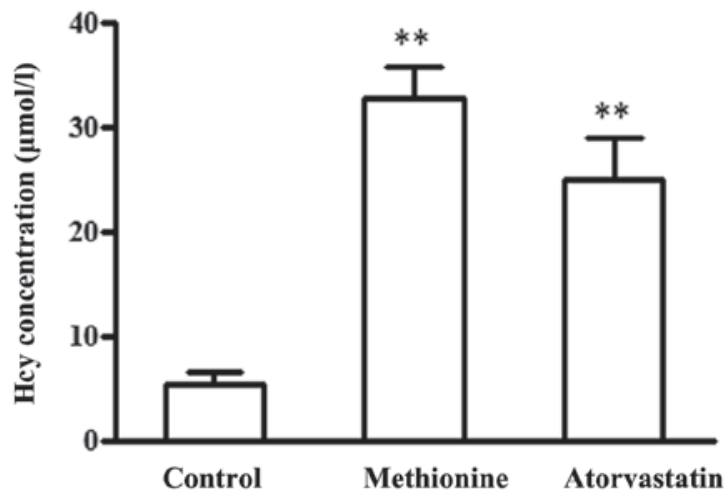

Figure 1. Plasma concentrations of homocysteine (Hcy) in the control, methionine and atorvastatin groups. Data are presented as the mean \pm standard deviation. ${ }^{* *} \mathrm{P}<0.01$ vs. the control group.

anticoagulated with ethylenediaminetetraacetic acid and placed on ice. Plasma was quickly separated by centrifugation at $1566 \mathrm{x} \mathrm{g}$ for $10 \mathrm{~min}$ at room temperature. The plasma was then stored at $-20^{\circ} \mathrm{C}$ until further analysis. Total Hcy concentrations were measured using high-performance liquid chromatography following reduction of the plasma samples (15).

Histological analysis. The aortic roots were carefully dissected and cleaned of adherent connective tissue under a dissecting microscope. The aortic roots were then embedded in paraffin and were serially sectioned at $4 \mu \mathrm{m}$. For quantification of atherosclerotic lesions, the sections were collected and stained with hematoxylin and eosin (H\&E) and Masson's trichrome (Sigma-Aldrich). Images of staining were captured and quantified using an Axioplan 2 imaging microscope system (Carl Zeiss GmbH, Jena, Germany).

Immunohistochemical (IHC) analysis. For IHC analysis, the paraffin-embedded sections were deparaffinized by immersion in xylene, followed by a series of alcohol treatments. Endogenous peroxidase activity was quenched by immersing the slides in $0.3 \%$ hydrogen peroxide in methanol for $15 \mathrm{~min}$. The sections were rinsed three times in PBS (5 min/wash) and were blocked with 5\% normal serum (Cell Signaling Technology, Inc., Danvers, MA, USA). The sections were then incubated with primary antibodies $(1: 1,000)$ overnight at $4^{\circ} \mathrm{C}$. Rabbit polyclonal phosphorylated (p)-protein kinase RNA-like endoplasmic reticulum kinase (PERK) antibodies were obtained from Santa Cruz Biotechnology, Inc. (Dallas, TX, USA; cat. no. sc-32577); rabbit monoclonal antibodies against glucose-regulated protein 78 (GRP78; cat. no. 3177) and p-eukaryotic initiation factor $2 \alpha$ (eIF $2 \alpha$; cat. no. 3398) were obtained from Cell Signaling Technology, Inc. Following rinsing in PBS with Tween 20, the sections were incubated with labeled polymer-horseradish peroxidase (HRP) goat anti-rabbit IgG (Cell Signaling Technology, Inc.; cat. no. 7074) at $37^{\circ} \mathrm{C}$ for $1 \mathrm{~h}$, and then incubated with diaminobenzidine chromogen. Following the final wash, the sections were counterstained with hematoxylin. The intensity of IHC staining was measured using the Axioplan 2 imaging analysis system (Carl Zeiss $\mathrm{GmbH}$ ). The average value in each group was calculated 
A

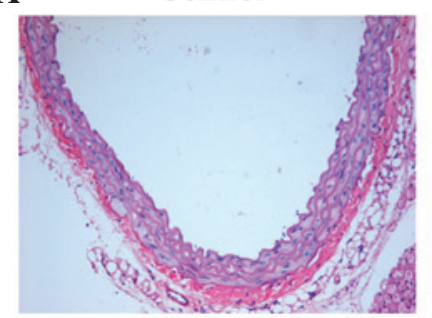

B

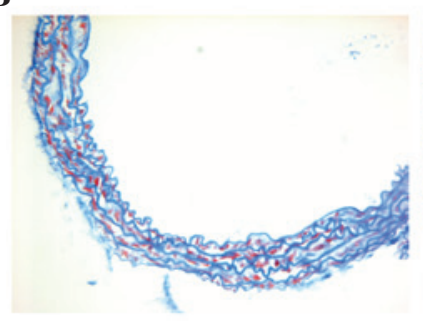

Methionine
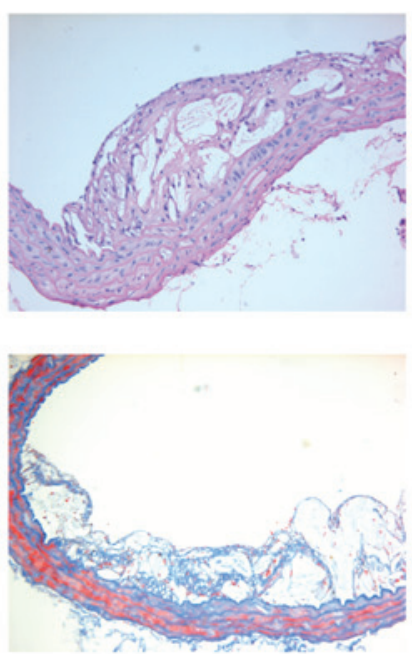

Atorvastatin
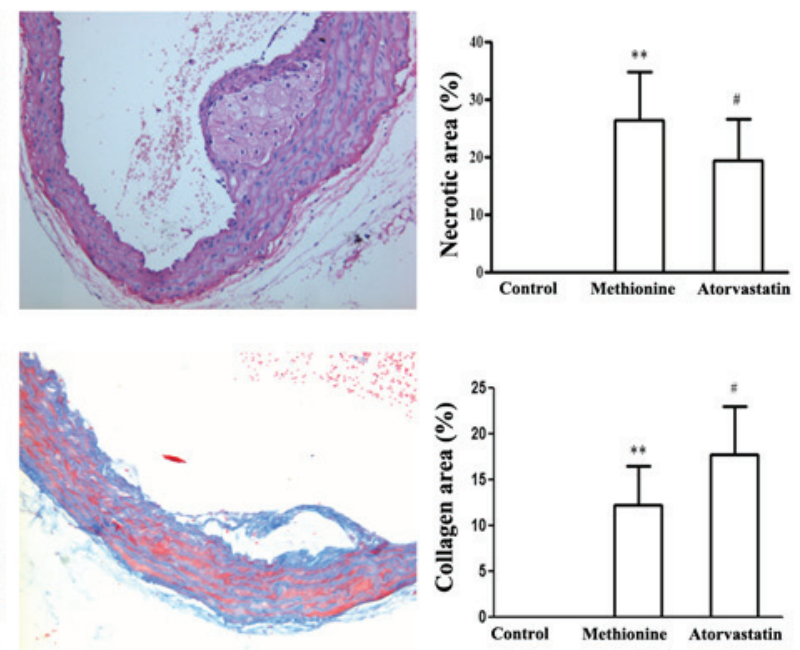

Figure 2. Effects of atorvastatin on plaque composition in the aortic roots of hyperhomocysteinemic apolipoprotein E-deficient mice. (A) Representative images of hematoxylin and eosin-stained aortas. (B) Representative images of Masson's trichrome-stained aortas. Magnification, x100. Data are presented as the mean \pm standard deviation. ${ }^{* *} \mathrm{P}<0.01$ vs. the control group; ${ }^{\#} \mathrm{P}<0.05$ vs. the methionine group.

from random observation of five high power microscopic views of entire sections.

Cell culture. The RAW264.7 murine macrophages were obtained from the Type Culture Collection of the Chinese Academy of Sciences (Shanghai, China). Cells were cultured in Dulbecco's modified Eagle's medium (Gibco; Thermo Fisher Scientific, Inc., Waltham, MA, USA) supplemented with $10 \%$ fetal bovine serum (Gibco; Thermo Fisher Scientific, Inc.) and $1 \%$ penicillin/streptomycin (Beyotime Institute of Biotechnology, Haimen, China). The cultures were maintained at $37^{\circ} \mathrm{C}$ in a humidified incubator containing $5 \% \mathrm{CO}_{2}$ until subconfluent. Cells received treatments in serum-free medium. A total of 1 and $10 \mu \mathrm{mol} / \mathrm{l}$ atorvastatin (National Institute for the Control of Pharmaceutical and Biological Products, Beijing, China), in the presence or absence of thapsigargin ( $2 \mu \mathrm{mol} / \mathrm{l}$; Sigma-Aldrich) was added $30 \mathrm{~min}$ prior to $500 \mu \mathrm{mol} / 1 \mathrm{Hcy}$ (Sigma-Aldrich) stimulation for $24 \mathrm{~h}$.

Reverse transcription-quantitative polymerase chain reaction (RT-qPCR) analysis. According to the manufacturer's protocol, RNA was extracted from macrophages or homogenized aortic roots using TRIzol ${ }^{\circledR}$ reagent (Invitrogen; Thermo Fisher Scientific, Inc.). The quality of the isolated RNA was determined using agarose gel electrophoresis, and RNA concentration was determined by measuring optical density at 260 and $280 \mathrm{~nm}$ using a BioPhotometer (Eppendorf, Hamburg, Germany). A reverse transcription kit (Takara Bio Inc., Otsu, Japan) was used for reverse transcription. The following primer sequences were designed (Invitrogen; Thermo Fisher Scientific, Inc.): TNF- $\alpha$, forward 5'-TTCTATGGCCCAGACCCTCA-3', reverse 5'-ACTTGGTGGTTTGCTACGACG-3'; MMP-9, forward 5'-GTCCCACTATACCTCCCACG-3', reverse 5'-ATT GCAAGGATTGTCTGCCG-3'; and $\beta$-actin, forward 5'-ATG GTGGGAATGGGTCAGAA-3' and reverse 5'-GTCACGCAC GATTTCCCTCT-3'. RT-qPCR was performed using SYBR ${ }^{\circledR}$ Premix Ex Taq ${ }^{\mathrm{TM}}$ (Perfect Real Time) kit (Takara Bio Inc., Otsu, Japan) and an Applied Biosystems 7300 Real-Time PCR

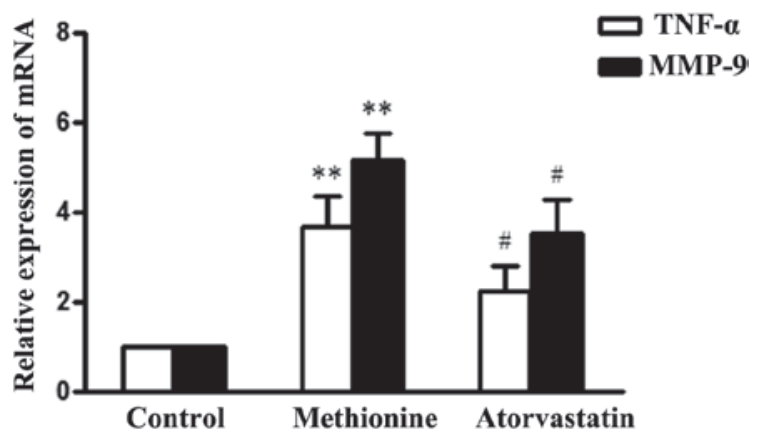

Figure 3. Effects of atorvastatin on tumor necrosis factor (TNF)- $\alpha$ and matrix metalloproteinase (MMP)-9 mRNA expression in the aortic roots of hyperhomocysteinemic apolipoprotein E-deficient mice. Data are presented as the mean \pm standard deviation. ${ }^{* *} \mathrm{P}<0.01$ vs. the control group; ${ }^{\#} \mathrm{P}<0.05$ vs. the methionine group.

system (Applied Biosystems; Thermo Fisher Scientific, Inc.). PCR was performed using an initial step of denaturation at $95^{\circ} \mathrm{C}$ for $30 \mathrm{sec}, 40$ cycles of amplification, denaturation at $95^{\circ} \mathrm{C}$ for $5 \mathrm{sec}$ and annealing at $60^{\circ} \mathrm{C}$ for $30 \mathrm{sec}$. All reactions were performed in a $20-\mu 1$ volume in triplicate. The relative amounts of mRNA in untreated and treated cells were compared using the comparative cycle quantification $\left(2^{\Delta \Delta \mathrm{Cq}}\right)$ method (16), with $\beta$-actin mRNA as the internal standard.

Western blot analysis. The cells were collected and radioimmunoprecipitation assay lysis buffer containing protease inhibitors (Beyotime Institute of Biotechnology) was used for extraction of total cellular protein. Protein concentrations were determined using a protein assay kit (Pierce Protein Biology; Thermo Fisher Scientific, Inc.). After boiling for $5 \mathrm{~min}$, equal quantities of the denatured protein sample $(40 \mu \mathrm{g})$ were separated by $9 \%$ sodium dodecyl sulfate-polyacrylamide gel electrophoresis and then protein was transferred electrophoretically to polyvinylidene fluoride membranes (Millipore, Bedford, MA, USA). The resulting membranes were blocked 


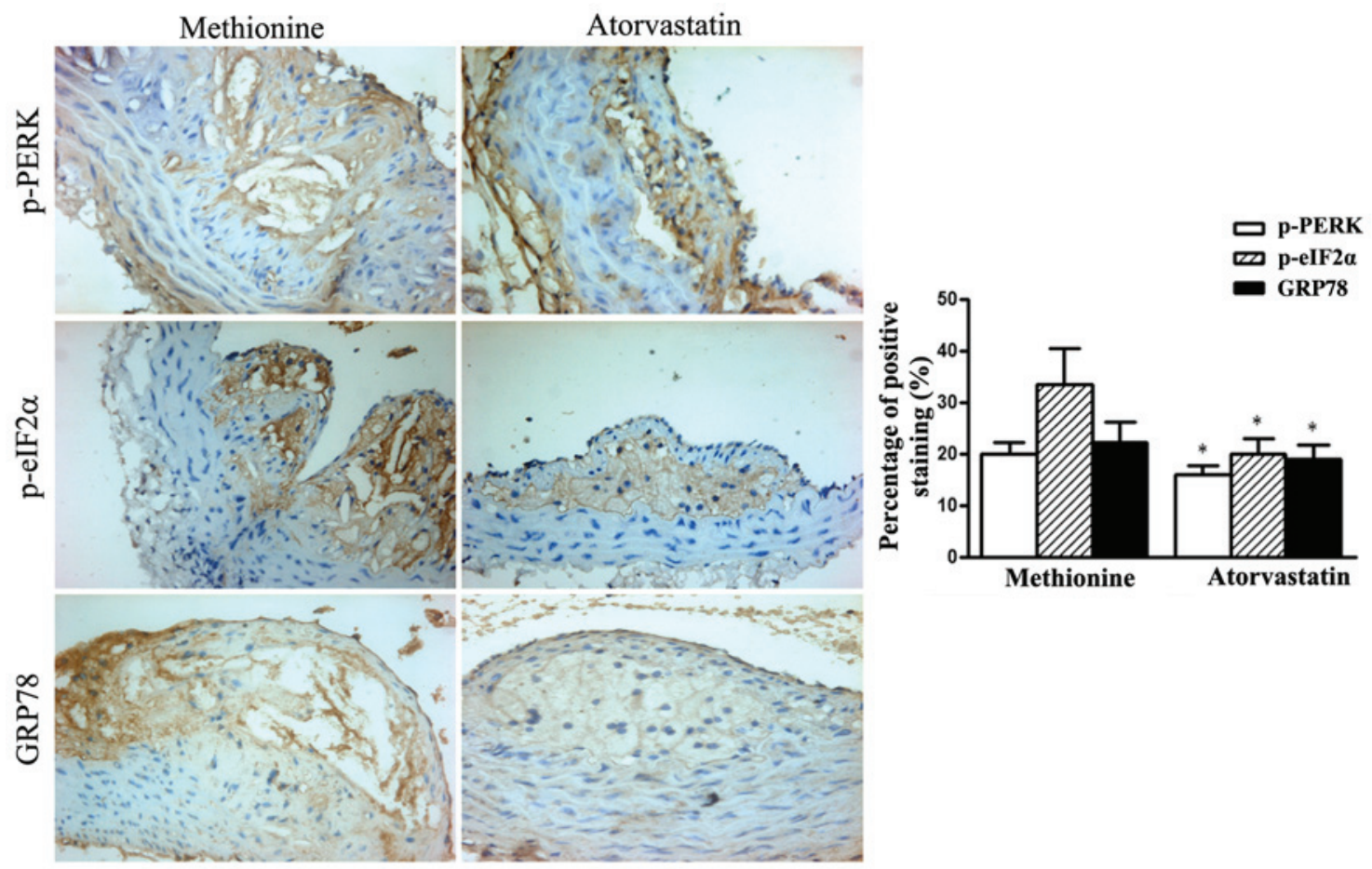

Figure 4. Effects of atorvastatin on endoplasmic reticulum stress activation in aortic lesions of hyperhomocysteinemic apolipoprotein E-deficient mice. Immunohistochemical analysis detected a significant decrease in phosphorylated (p)-protein kinase RNA-like endoplasmic reticulum kinase (PERK), p-eukaryotic initiation factor $2 \alpha$ (eIF2 $\alpha$ ) and glucose-regulated protein 78 (GRP78) immunostaining in the atorvastatin group, as compared with the positive staining of these markers in the methionine group. Magnification, $\mathrm{x} 200$. Data are presented as the mean \pm standard deviation. ${ }^{*} \mathrm{P}<0.05$ vs. the methionine group.

using 5\% non-fat milk in Tris-buffered saline containing Tween (10 mmol/1 Tris-HCl, pH 7.4; $150 \mathrm{mmol} / 1 \mathrm{NaCl} ; 0.05 \%$ Tween-20) for $1 \mathrm{~h}$, and hybridized with primary antibodies (antibody against p-PERK, 1:400 dilution; cat. no. sc-32577; Santa Cruz Biotechnology, Inc.; antibodies against GRP78 and p-eIF2 $\alpha$; cat. nos. 3177 and 3398; Cell Signaling Technology, Inc.; 1:1,000 dilution) overnight at $4^{\circ} \mathrm{C}$. Following incubation with the appropriate HRP-conjugated goat anti-rabbit IgG secondary antibody (1:1,000 dilution; cat. no. 7074; Cell Signaling Technology, Inc.), the immune complexes were detected using an enhanced chemiluminescence detection system (GE Healthcare Life Sciences, Chalfont, UK). Staining was quantified by scanning densitometry (Microtek Scanwizard 5; Informer Technologies, Inc., Walnut, CA, USA) with $\beta$-actin used as an internal standard.

Statistical analysis. The obtained data are presented as the mean \pm standard deviation. One-way analysis of variance was used to statistically analyze the data, using SPSS 13.0 software (SPSS, Inc., Chicago, IL, USA). P<0.05 (two-tailed) was considered to indicate a statistically significant difference.

\section{Results}

Effects of atorvastatin on plasma Hcy levels in ApoE $E^{-/}$mice. The methionine group were administered $1 \mathrm{ml} 2 \%(\mathrm{w} / \mathrm{v})$ methionine, and the atorvastatin group were administered atorvastatin $\left(5 \mathrm{mg} \cdot \mathrm{kg}^{-1} \cdot \mathrm{d}^{-1}\right)$ suspended in $1 \mathrm{ml} 2 \%$ methionine daily for 2 months. After 2 months, plasma Hcy levels in the methionine group were significantly increased, as compared with in the control ApoE ${ }^{-/-}$mice $(32.8 \pm 3.0$ vs. $5.3 \pm 1.2 \mu \mathrm{mol} / 1$,
$\mathrm{P}<0.01)$. In addition, plasma Hcy levels were upregulated in the atorvastatin group $(25.0 \pm 3.9 \mu \mathrm{mol} / \mathrm{l})$ compared with the control group; however, there was no statistical difference between the atorvastatin and methionine groups (Fig. 1).

Atorvastatin inhibits vulnerable plaque formation in the aortic roots of HHcy mice. The present study analyzed large necrotic core area and collagen content, in order to investigate plaque stability. Paraffin sections from the aortic roots of $\mathrm{ApoE}^{-/-}$mice were stained with $\mathrm{H} \& \mathrm{E}$ to assess lesion growth and histological morphology (Fig. 2A). To determine whether atorvastatin was able to reduce necrotic core formation, both the number and the relative size of necrotic cores was analyzed. Treatment with atorvastatin resulted in fewer necrotic cores, as compared with the methionine group; $30.83 \pm 7.91$ vs. $44.2 \pm 13.12 \%$ of the sections covering the entire lesion that contained a necrotic core $(\mathrm{P}<0.05)$. Furthermore, the relative size of the necrotic cores decreased from $26.42 \pm 8.23$ to $19.41 \pm 7.05 \%$ of the plaque surface area $(\mathrm{P}<0.05)$. Collagen is the main stabilizing component of plaques. Masson's trichrome staining detected a $45 \%$ increase in the relative amount of collagen in the plaques following atorvastatin treatment, as compared with that in the methionine group $(17.75 \pm 5.25$ vs. $12.21 \pm 4.28 \%, \mathrm{P}<0.05)$ (Fig. 2B).

Atorvastatin reduces TNF- $\alpha$ and MMP-9 mRNA expression in the aortic roots of HHcy mice. To explore the effects of atorvastatin on the HHcy-induced inflammatory response in aortic roots of $\mathrm{ApoE}^{-/-}$mice, TNF- $\alpha$ and MMP-9 mRNA expression levels were detected by RT-qPCR. TNF- $\alpha$ and MMP-9 expression levels were significantly upregulated in the methionine 

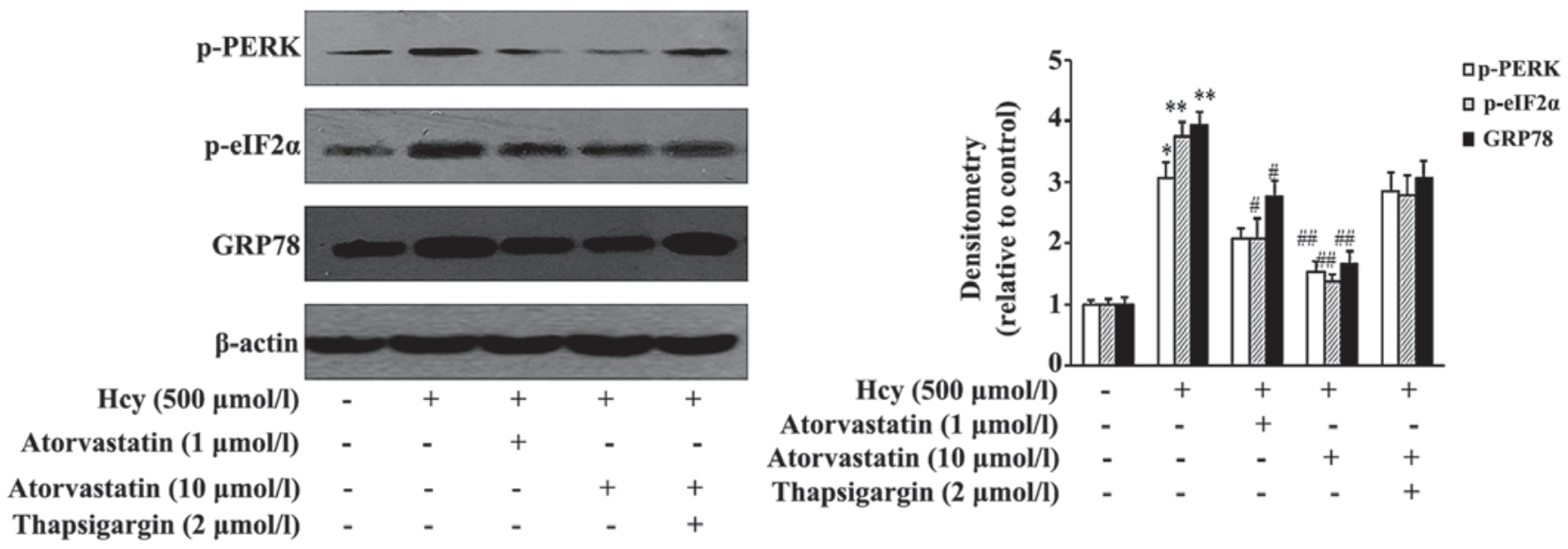

Figure 5. Effects of atorvastatin on homocysteine (Hcy)-induced endoplasmic reticulum stress in macrophages. Data are presented as the mean \pm standard deviation. ${ }^{*} \mathrm{P}<0.05$ and ${ }^{* *} \mathrm{P}<0.01$ vs. the control group; ${ }^{*} \mathrm{P}<0.05$ and ${ }^{\# \#} \mathrm{P}<0.01$ vs. the Hcy group. $\mathrm{p}$-, phosphorylated; PERK, protein kinase RNA-like endoplasmic reticulum kinase; eIF2 $\alpha$, eukaryotic initiation factor $2 \alpha$; GRP78, glucose-regulated protein 78.

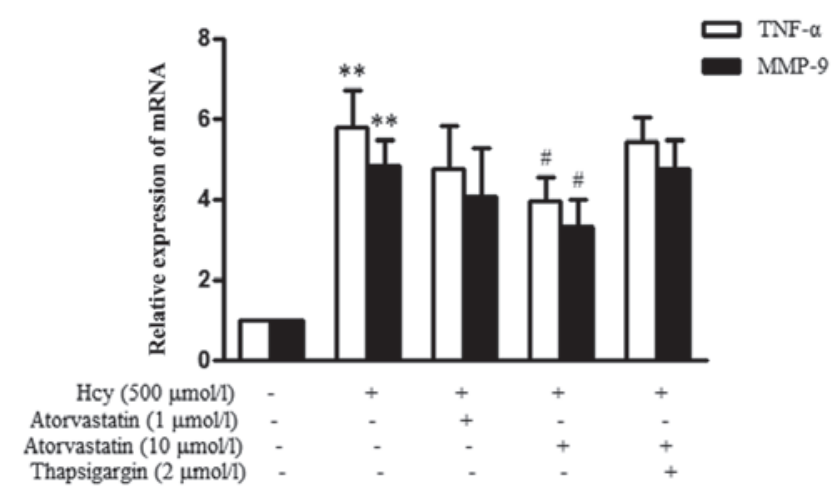

Figure 6. Effects of atorvastatin on homocysteine (Hcy)-induced tumor necrosis factor (TNF)- $\alpha$ and matrix metalloproteinase (MMP)-9 mRNA expression levels in macrophages. Data are presented as the mean \pm standard deviation. ${ }^{* *} \mathrm{P}<0.01$ vs. the control group; ${ }^{*} \mathrm{P}<0.05$ vs. the Hcy group.

group, as compared with the control group, whereas treatment with atorvastatin significantly decreased the expression levels in atherosclerotic lesions (Fig. 3).

Atorvastatin prevents ER stress activation in aortic lesions of HHcy ApoE ${ }^{-/-}$mice. To further determine the mechanisms underlying statin-induced plaque stabilization, the present study investigated whether atorvastatin affected HHcy-induced ER stress in aortic root lesions (Fig. 4). The IHC analysis detected a significant decrease in p-PERK immunostaining in the atorvastatin group, as compared with in the methionine group. Furthermore, p-eIF2 $\alpha$ and GRP78 immunostaining were significantly downregulated following treatment with atorvastatin, as compared with following methionine treatment only. These results indicate that atorvastatin may inhibit ER stress activation in aortic lesions of HHcy mice. The plaque stabilizing effects of atorvastatin may be due to ER stress inhibition in HHcy mice.

Atorvastatin inhibits Hcy-induced ER stress in macrophages. To determine the effects of atorvastatin on Hcy-induced ER stress in macrophages, various concentrations of atorvastatin ( 1 and $10 \mu \mathrm{mol} / \mathrm{l})$ were added $30 \mathrm{~min}$ prior to $500 \mu \mathrm{mol} / 1 \mathrm{Hcy}$ stimulation. As shown in Fig. 5, $500 \mu \mathrm{mol} / \mathrm{l}$ Hcy induced ER stress activation in macrophages, as determined by the increased phosphorylation of PERK and its substrate, eIF2 $\alpha$, and the increased expression of the chaperone GRP78. Compared with the Hcy group, Hcy-induced ER stress was inhibited in response to $10 \mu \mathrm{mol} / 1$ atorvastatin treatment. Furthermore, thapsigargin, an ER stress inducer, attenuated the inhibitory effects of atorvastatin against Hcy-induced ER stress.

Atorvastatin suppresses the Hcy-induced inflammatory response in macrophages. The present study also evaluated the effects of atorvastatin on Hcy-induced TNF- $\alpha$ and MMP-9 mRNA expression. As shown in Fig. 6, the relative mRNA expression levels of TNF- $\alpha$ were significantly upregulated in response to Hcy stimulation. However, treatment with $10 \mu \mathrm{mol} / 1$ atorvastatin could evidently inhibit Hcy-induced TNF- $\alpha$ mRNA expression. MMP-9 expression was also significantly upregulated in the Hcy group, as compared with in the control group, whereas treatment with $10 \mu \mathrm{mol} / 1$ atorvastatin significantly decreased its expression. Furthermore, thapsigargin attenuated the inhibitory effects of atorvastatin against Hcy-induced TNF- $\alpha$ and MMP-9 upregulation. These results strongly suggest that ER stress pathways may participate in the reaction, and atorvastatin inhibits the Hcy-induced inflammatory response via suppressing ER stress in macrophages.

\section{Discussion}

The present study demonstrated that HHcy promoted the development of atherosclerotic plaques and plaque instability. Atorvastatin was able to downregulate Hcy-induced ER stress activation in atherosclerotic lesions and macrophages, which may suppress inflammatory responses and improve the stability of atherosclerotic plaques.

Alterations in atherosclerotic plaque composition can impact plaque stability, and determine the clinical course of atherosclerosis. Previous studies have suggested that the inflammatory cytokines have an important role 
in the disruption of vascular function, and the resultant development of vascular diseases $(17,18)$. In addition, epidemiological studies have reported that TNF- $\alpha$ is markedly elevated in the plasma and arteries of human patients with vascular complications $(19,20)$. Elevated circulating levels of matrix biomarkers are a key feature of atherosclerotic plaque development, vascular remodeling and plaque rupture. MMP-9, which contributes to the formation of unstable plaques, has been suggested as an atherosclerotic inflammatory marker (21). In the present study, methionine treatment enabled the generation of a HHcy atherosclerotic $\mathrm{ApoE}^{-/-}$mouse model. Atherosclerotic plaques in the aortic arteries of the mice appeared to possess several key histological features of unstable plaques, including large necrotic cores, reduced collagen content and increased inflammatory cytokine infiltration.

The pathophysiological environment in HHcy mice was able to activate prolonged ER stress in the arterial wall. The present study demonstrated that the expression of p-PERK, p-eIF2 $\alpha$ and GRP78 were predominantly situated within the atherosclerotic lesions in $\mathrm{HHcy} \mathrm{ApoE}^{-/}$mice. Previous studies have revealed that ER stress is a cross-point that links cellular processes with numerous risk factors that exist in all stages of atherosclerosis, and is believed to have a critical role in endothelial dysfunction $(22,23)$, activation of inflammatory reactions (24) and foam cell formation (25). Activation of ER stress is associated with the severity and clinical complications of atherosclerosis in humans. In a previous study, directional coronary atherectomy specimens demonstrated that ruptured plaques exhibited a markedly increased expression of the ER chaperones GRP78 and CCAAT-enhancer-binding protein homologous protein (CHOP) (26). These findings further supported the hypothesis that vulnerability of artery plaques in HHcy mice may be partially attributed to ER stress activation.

Therapeutic interventions that reduce ER stress may be considered promising strategies to reduce Hcy-induced vascular injury and consequent plaque instability. In the present study, administration of atorvastatin to $\mathrm{ApoE}^{-/-}$treated with methionine resulted in a decreased number and size of necrotic cores, and increased collagen content in the plaques, as compared with the plaques in the methionine group. Downregulation of TNF- $\alpha$ and MMP-9 in the lesions of the atorvastatin-treated mice further substantiated these findings. Atorvastatin is widely used in the treatment and prevention of atherosclerosis. Atorvastatin has previously been shown to downregulate GRP78, caspase-12 and CHOP expression in myocardial cells (27), and attenuate myocardial ischemia reperfusion injury via inhibiting ER stress-related apoptosis (28). Atorvastatin may function as a pharmacological inhibitor of ER stress. In the present study, treatment with atorvastatin significantly suppressed ER stress activation and reduced plaque vulnerability, thus indicating that atorvastatin may maintain ER homeostasis and prevent HHcy-induced atherosclerotic lesion progression. However, atorvastatin did not reduce plasma Hcy levels in $\mathrm{ApoE}^{-/-}$mice, thus indicating that the plaque stabilizing effects of atorvastatin against Hcy injury may not depend on the lowering of Hcy levels. Inhibition of ER stress may serve as an important role in the plaque stabilizing effects of atorvastatin.
The present study also demonstrated that atorvastatin could suppress Hcy-induced ER stress activation in macrophages. Macrophage infiltration plays a crucial role throughout the entire process of atherogenesis and plaque rupture. Cytokines secreted by macrophages, including MMPs and TNF- $\alpha$, can attenuate plaque stability and prompt rupture of atherosclerotic plaques. In the present study, treatment with Hcy increased MMP-9 and TNF- $\alpha$ expression in murine macrophages; however, Hcy-induced MMP-9 and TNF- $\alpha$ mRNA expression was markedly attenuated by atorvastatin. Thapsigargin attenuated the protective effects of atorvastatin against Hcy-induced ER stress and MMP-9 and TNF- $\alpha$ production, thus suggesting that ER stress pathways have predominant roles in Hcy-induced inflammation in macrophages. Atorvastatin may inhibit the Hcy-induced inflammatory response via suppressing ER stress in macrophages.

In conclusion, the results of the present study provide a novel insight into the protective effects of atorvastatin against Hcy-induced vascular injury. Hcy markedly promoted the development of atherosclerotic plaques and plaque instability. Atorvastatin was used to antagonize Hcy-induced injury in HHcy ApoE ${ }^{-/-}$mice and in macrophages, and it was shown to target ER molecules and inhibit the development of atherosclerotic lesions. Atorvastatin may therefore attenuate the progression of atherosclerotic lesions and plaque vulnerability by regulating ER stress activation, which may provide a novel interpretation of its pleiotropic effects. However, further studies are required to fully understand the relationship between atorvastatin and ER stress activation in the treatment of atherosclerosis.

\section{Acknowledgements}

The present study was supported by the National Natural Science Foundation of China (grant no. 81300220).

\section{References}

1. Silvestre-Roig C, de Winther MP, Weber C, Daemen MJ, Lutgens E and Soehnlein O: Atherosclerotic plaque destabilization: Mechanisms, models, and therapeutic strategies. Circ Res 114: 214-226, 2014.

2. Hopkins PN: Molecular biology of atherosclerosis. Physiol Rev 93: 1317-1542, 2013.

3. Gautier EL, Huby T, Witztum JL, Ouzilleau B, Miller ER, Saint-Charles F, Aucouturier P, Chapman MJ and Lesnik P: Macrophage apoptosis exerts divergent effects on atherogenesis as a function of lesion stage. Circulation 119: 1795-1804, 2009.

4. Tabas I: The role of endoplasmic reticulum stress in the progression of atherosclerosis. Circ Res 107: 839-850, 2010.

5. Yao S, Miao C, Tian H, Sang H, Yang N, Jiao P, Han J, Zong C and Qin S: Endoplasmic reticulum stress promotes macrophage-derived foam cell formation by up-regulating cluster of differentiation 36 (CD36) expression. J Biol Chem 289: 4032-4042, 2014.

6. Gao J, Ishigaki Y, Yamada T, Kondo K, Yamaguchi S, Imai J, Uno K, Hasegawa Y, Sawada S, Ishihara H, et al: Involvement of endoplasmic stress protein $\mathrm{C} / \mathrm{EBP}$ homologous protein in arteriosclerosis acceleration with augmented biological stress responses. Circulation 124: 830-839, 2011.

7. McAlpine CS, Bowes AJ, Khan MI, Shi Y and Werstuck GH: Endoplasmic reticulum stress and glycogen synthase kinase- $3 \beta$ activation in apolipoprotein E-deficient mouse models of accelerated atherosclerosis. Arterioscler Thromb Vasc Biol 32: 82-91, 2012. 
8. Zhou J and Austin RC: Contributions of hyperhomocysteinemia to atherosclerosis: Causal relationship and potential mechanisms. Biofactors 35: 120-129, 2009.

9. Qiu G and Hill JS: Atorvastatin inhibits ABCA1 expression and cholesterol efflux in THP-1 macrophages by an LXR-dependent pathway. J Cardiovasc Pharmacol 51: 388-395, 2008.

10. Llaverias G, Noé V, Peñuelas S, Vázquez-Carrera $M$, Sánchez RM, Laguna JC, Ciudad CJ and Alegret M: Atorvastatin reduces CD68, FABP4, and HBP expression in oxLDL-treated human macrophages. Biochem Biophys Res Commun 318: 265-274, 2004

11. Gómez-Hernández A, Sánchez-Galán E, Martín-Ventura JL, Vidal C, Blanco-Colio LM, Ortego M, Vega M, Serrano J, Ortega L, Hernández $\mathrm{G}$, et al: Atorvastatin reduces the expression of prostaglandin E2 receptors in human carotid atherosclerotic plaques and monocytic cells: Potential implications for plaque stabilization. J Cardiovasc Pharmacol 47: 60-69, 2006

12. Jia F, Wu C, Chen $\mathrm{Z}$ and Lu G: AMP-activated protein kinase inhibits homocysteine-induced dysfunction and apoptosis in endothelial progenitor cells. Cardiovasc Drugs Ther 25: 21-29, 2011.

13. Bao XM, Wu CF and Lu GP: Atorvastatin attenuates homocysteine-induced apoptosis in human umbilical vein endothelial cells via inhibiting NADPH oxidase-related oxidative stress-triggered p38MAPK signaling. Acta Pharmacol Sin 30: 1392-1398, 2009.

14. National Research Council (US) Committee for the Update of the Guide for the Care and Use of Laboratory Animals: Guide for the Care and Use of Laboratory Animals. 8th edition. National Institutes of Health, The National Academies Press, Washington, DC, 2011

15. Jacobsen DW, Gatautis VJ, Green R, Robinson K, Savon SR, Secic M, Ji J, Otto JM and Taylor LM Jr: Rapid HPLC determination of total homocysteine and other thiols in serum and plasma: Sex differences and correlation with cobalamin and folate concentrations in healthy subjects. Clin Chem 40 873-881, 1994

16. Livak KJ and Schmittgen TD: Analysis of relative gene expression data using real-time quantitative PCR and the 2(-Delta Delta C(T)) Method. Methods 25: 402-408, 2001.

17. Shah PK: Molecular mechanisms of plaque instability. Curr Opin Lipidol 18: 492-499, 2007.
18. Cavieres V, Valdes K, Moreno B, Moore-Carrasco R and Gonzalez DR: Vascular hypercontractility and endothelial dysfunction before development of atherosclerosis in moderate dyslipidemia: Role for nitric oxide and interleukin-6. Am J Cardiovasc Dis 4: 114-122, 2014.

19. McLaren JE, Michael DR, Ashlin TG and Ramji DP: Cytokines, macrophage lipid metabolism and foam cells: Implications for cardiovascular disease therapy. Prog Lipid Res 50: 331-347, 2011.

20. Vizzardi E, Cavazzana I, Sciatti E, Bonadei I, D'Aloia A, Tincani A, Franceschini $\mathrm{F}$ and Metra M: Evaluation of ascending aorta wall in rheumatoid arthritis by tissue and strain Doppler imaging during anti-tumor necrosis factor- $\alpha$ therapy. Clin Cardiol 37: 738-743, 2014.

21. Lim HS and Lip GY: Circulating matrix metalloproteinase-9 levels in atherosclerotic vascular disease: A possible measurement of systemic or specific disease pathophysiology? J Intern Med 263: 620-622, 2008

22. Galán M, Kassan M, Kadowitz PJ, Trebak M, Belmadani S and Matrougui K: Mechanism of endoplasmic reticulum stress-induced vascular endothelial dysfunction. Biochim Biophys Acta 1843: 1063-1075, 2014.

23. Civelek M, Manduchi E, Riley RJ, Stoeckert CJ Jr and Davies PF: Chronic endoplasmic reticulum stress activates unfolded protein response in arterial endothelium in regions of susceptibility to atherosclerosis. Circ Res 105: 453-461, 2009.

24. Zhou AX and Tabas I: The UPR in atherosclerosis. Semin Immunopathol 35: 321-332, 2013.

25. Oh J, Riek AE, Weng S, Petty M, Kim D, Colonna M, Cella M and Bernal-Mizrachi C: Endoplasmic reticulum stress controls M2 macrophage differentiation and foam cell formation. J Biol Chem 287: 11629-11641, 2012.

26. Myoishi M, Hao H, Minamino T, Watanabe K, Nishihira K, Hatakeyama K, Asada Y, Okada K, Ishibashi-Ueda H, Gabbiani G, et al: Increased endoplasmic reticulum stress in atherosclerotic plaques associated with acute coronary syndrome. Circulation 116: 1226-1233, 2007.

27. Song XJ, Yang CY, Liu B, Wei Q, Korkor MT, Liu JY and Yang P: Atorvastatin inhibits myocardial cell apoptosis in a rat model with post-myocardial infarction heart failure by downregulating ER stress response. Int J Med Sci 8: 564-572, 2011.

28. Xia JG, Xu FF, Qu Y, Song DG, Shen H and Liu XH: Atorvastatin post-conditioning attenuates myocardial ischemia reperfusion injury via inhibiting endoplasmic reticulum stress-related apoptosis. Shock 42: 365-371, 2014. 